“(C) 2019 IEEE. Personal use of this material is permitted. Permission from IEEE must be obtained for all other uses, in any current or future media, including reprinting/republishing this material for advertising or promotional purposes, creating new collective works, for resale or redistribution to servers or lists, or reuse of any copyrighted component of this work in other works." 


\section{Opportunities for a Cloud based Health Analytics as a Service for Eastern Cape Initiation Schools in South Africa}

\author{
Priscilla, Xoliswa Majola \\ Graduate School of Business \& Leadership \\ University of Kwazulu-Natal \\ Durban, South Africa \\ Majolax@ukzn.ac.za
}

\author{
Carolyn McGregor \\ Faculty of Business and IT \\ Ontario Tech University, Oshawa, Canada \\ University of Technology Sydney, Sydney, Australia \\ c.mcgregor@ieee.org
}

\begin{abstract}
Traditional male circumcision in the contemporary South Africa has become the focus of the government and media due to the large number of initiates severely injured or dying during the initiation period, which happens twice a year. Deaths and penile amputations are a feature of every circumcision season, as a result of sepsis, gangrene and dehydration amongst other diseases. This paper proposes a Cloud based Health Analytics as a Service for Eastern Cape initiation schools in South Africa to assist in saving lives and preserving the customs. The proposed Artemis platform will assist in acquiring physiological data of initiates before and during initiation to provide early insights of many conditions that can develop during initiation. Big data analytics based on Clinical Decision Support System such as Artemis provides real-time online analytics with knowledge extraction component that supports data mining and enables clinical research of various conditions. Conversely, Artemis has challenges for lower resource settings, which will be explored in this paper.
\end{abstract}

Keywords-Big Data, Initiation Schools, Cloud Computing, Physiological Monitoring

\section{INTRODUCTION}

Male circumcision is universally common to many cultures across the world. There are different types or forms of how it is practiced. In South Africa, it is regarded as a rite of passage acknowledging the initiation of an individual into a group or society. This tradition is the most common amongst the Xhosa ethnic group, which predominantly resides in the province of Eastern Cape of South Africa. It is used "as a transitional rite of passage from 'boyhood to manhood', conferring on the individual the right to participate in the decision-making processes of the clan and family; to share in the privileges, duties and responsibilities of the community and, in many instances, to take a wife and raise a family" [1].

The authors propose the use of Artemis Cloud framework as a platform Health Analytics-as a-Service (HAaaS) model. This model will allow collection of initiates' physiological data before and during initiation [2]. Acquisition of physiological data streams presents great potential for early detection of critical conditions amongst patients [2, 3]. A new platform is needed to enable real-time analysis of physiological data from several data streams to support screening for multiple conditions of multiple initiates at different locations.
Artemis is a Big Data analytics computing platform for Clinical Decision Support Systems. It enables the integrated analysis of multiple physiological data streams from multiple patients for earlier onset detection of a range of clinical conditions [2]. This has great potential to provide earlier indicators of health decline to healthcare professionals, traditional healers and nurses.

\section{RELATED WORK}

Big Data has delivered a cost-effective prospect for improving decision-making in areas such as healthcare, economic productivity and security. Patient-centred model focuses on individual needs and preferences and healthcare informatics has contributed in the shift from population-based healthcare to patient-centred decision-making through using advanced analytics [4]. Big data is a multi-step process which includes acquisition, information extraction and cleaning, data extraction modelling and analysis, interpretation and deployment [5]. This paper proposes the use of big data as an intervention to traditional male circumcision. It is noted that in South Africa there is a lack of national guidance on big data storage and sharing, as well as a lack of framework to support clinical research [6].

\section{HeAlth Risks During TRAditional CiRCUMCISION}

In recent years, in South Africa, botched circumcisions by untrained and unscrupulous practitioners have increased, resulting in genital mutilation and often, the need for penile amputation [7]. Mortality rates during and after initiation clearly demonstrate the negligence of traditional surgeons and guardians, who are the most important men during initiation period and play a vital role in successful healing of initiate's wounds. Recent statistics that are available are for 2013 and only reflects the first season of initiation - June, while December data is not available. Notably, the statistics represent only the reported cases, while others remain unknown due to lack of disclosure by authorities and/or families [8]. 
Table 1. District Statistics of Traditional Male Circumcision for June 2013 in the Eastern Cape Province [9].

\begin{tabular}{|l|c|c|c|c|c|}
\hline District & $\begin{array}{c}\text { Legal } \\
\text { initiates }\end{array}$ & $\begin{array}{c}\text { Illegal } \\
\text { initiate } \\
\mathrm{s}\end{array}$ & $\begin{array}{c}\text { Number of } \\
\text { hospital } \\
\text { admissions }\end{array}$ & $\begin{array}{c}\text { Amputatio } \\
\text { ns of penis }\end{array}$ & $\begin{array}{c}\text { Number of } \\
\text { deaths }\end{array}$ \\
\hline $\begin{array}{l}\text { OR } \\
\text { Tambo }\end{array}$ & 5120 & 1120 & 259 & 24 & 26 \\
\hline $\begin{array}{l}\text { Chris } \\
\text { Hani }\end{array}$ & 1818 & 5 & 21 & 0 & 1 \\
\hline $\begin{array}{l}\text { Alfred } \\
\text { Nzo }\end{array}$ & 2323 & 1174 & 41 & 0 & 9 \\
\hline $\begin{array}{l}\text { Buffalo } \\
\text { City }\end{array}$ & 852 & 5 & 2 & 0 & 1 \\
\hline $\begin{array}{l}\text { Cacadu } \\
\text { Nelson } \\
\text { Mandel } \\
\text { a Bay }\end{array}$ & 501 & 0 & 0 & 0 & 0 \\
\hline $\begin{array}{l}\text { Joe } \\
\text { Gqabi }\end{array}$ & 265 & 2 & 13 & 0 & 1 \\
\hline $\begin{array}{l}\text { Amath } \\
\text { ole }\end{array}$ & 1084 & 8 & 23 & 0 & 2 \\
\hline $\begin{array}{l}\text { TOTA } \\
\text { L }\end{array}$ & 12169 & 2314 & 359 & 24 & 40 \\
\hline
\end{tabular}

The initiate's body dehydrates because it's unable to handle exposure to the sun or cold all day long [8]. Initiates become significantly dehydrated during their first 2 weeks of seclusion in the belief that this would reduce leakage of the wound. Factors that contribute to dehydration include deliberate restriction of intake of fluids and minimal or inadequate shelter, especially during summer, which can lead to increased sweating [10]. In addition, severe dehydration has been linked to renal failure.

Other causes of deaths that are linked to complications of traditional male circumcision include septicemia, gangrene, pneumonia, assault, thromboembolism, and congestive heart failure [10]. Prior recommendations have encouraged collaboration of health authorities and tribal groups to mitigate the complications of this culturally-driven circumcision. This then calls for a platform that will provide data acquisition, data transmission, real-time data analytics, data persistence, and retrospective knowledge discovery for initiates' physiological data with the tools used to extract, store, and analyse the data collected.

\section{ARTEMIS}

Artemis is a real-time Big Data platform that enables concurrent multi-participant, multi diagnosis and multi stream temporal analysis [8].

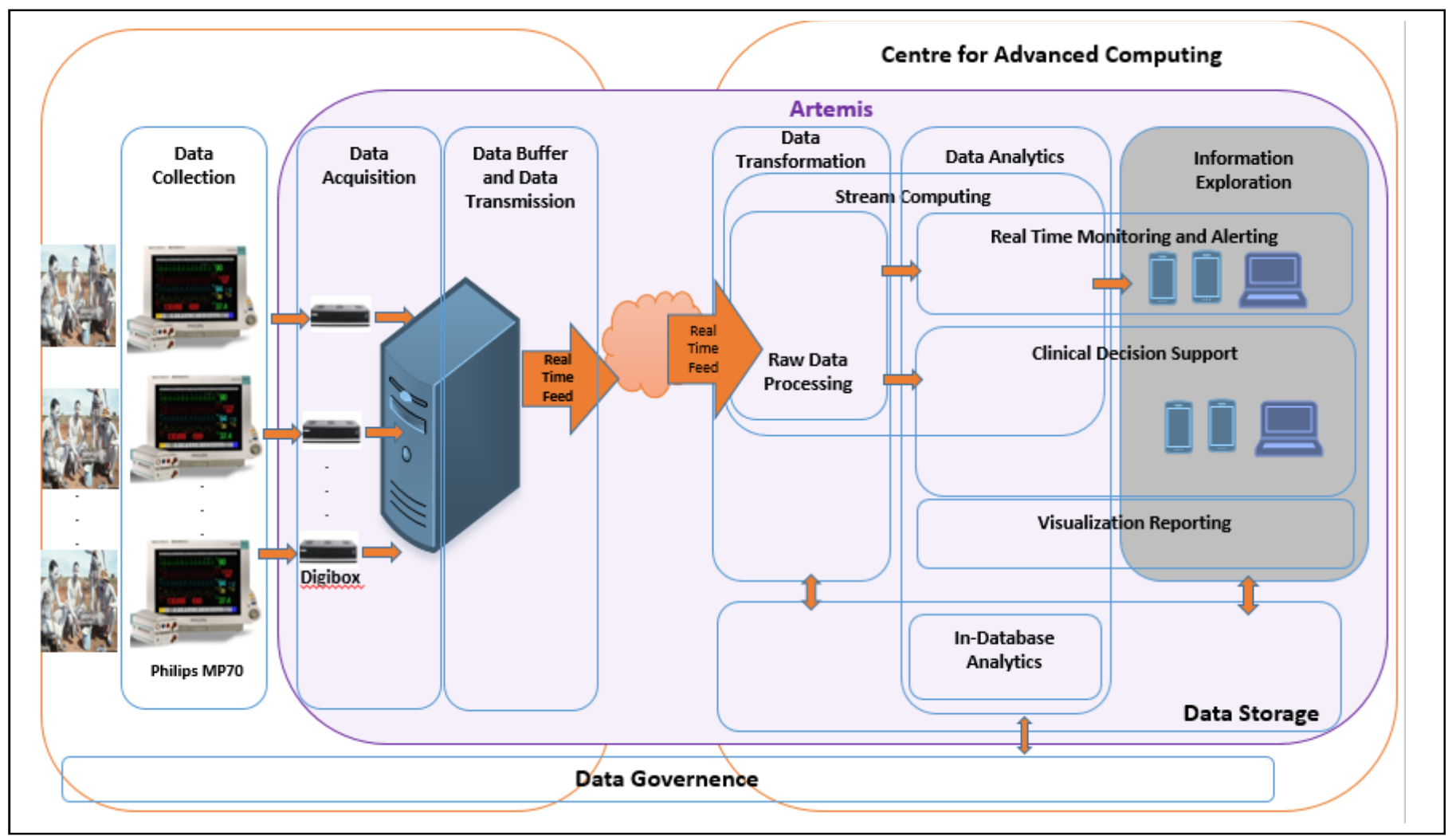

Fig. 1. Artemis Framework modified from [11]

Artemis was designed to provide a systematic framework instantiated through a platform to support Big Data analytics in critical care medicine [12]. The important components for the Artemis are: Data Acquisition which allows the collection of real-time synchronous medical device data and asynchronous Clinical Information Management System (CIMS) data. Data accumulated is then analyzed within the Online Analysis platform using IBMs InfoSphere Streams that 
operates in real-time. Data is stored within the Data Persistency component. Artemis has demonstrated the ability to process and store the raw data and data resulting from the temporal analysis from multiple patients at the speed the medical devices create the data [7]. Another Artemis component is the Knowledge Extraction component that utilizes McGregor's temporal data mining approach [8].

\section{ARTEMIS CLOUD}

The Artemis Cloud and its Health Analytics-as-a-Service (HAaaS) model determines how the new form of analytics can be provided as a service which will relieve the healthcare organisations from having to support such a platform in house [13]. A cloud-based software-as-a-service model proposed by McGregor [2] employs various web-based services that can allow hospitals to interact effectively with Artemis. Subsequently, Artemis Cloud has the ability to provide an interface for remote hospitals, which is supported through its Software-as-a-Service approach and also allows storage of persistent data through the Data-as-a-Service approach. The Artemis platform was successfully deployed in the the neonatal intensive care unit (NICU) at SickKids Hospital in Toronto in 2009 [2].

Traditional male circumcision can benefit from a Big Data approach such as Artemis that would exist for use by initiates and will include the following components:

\section{A. Data Acquisition - initiates}

The Artemis platform will be extended to support online health analytics during traditional male circumcision. This form of data acquisition will address an unmet demand to design a mechanism for the acquisition of physiological data from initiates on a regular basis something that has never been performed before. As a cultural design constraint, this data acquisition must not interfere with the tradition and also not hinder the various activities that happen during the initiation.

The Artemis platform allows the sourcing of data from a number of medical devices that are able to transmit the signal via wired or wireless connection. This will be best suited to process real-time data acquired from initiates. This will also allow for collection of other relevant clinical data related to initiates such as age, height and weight.

\section{B. Data Transmission - initiates}

Low resourced settings vary from well-resourced settings of the Western countries and this has consequences in this setting [2]. In this case, data transmission will require internet connection which will require introduction of cell towers and other means to provide internet in these secluded areas where temporal accommodations for initiates are placed. There will also be a need for electricity power for the monitoring devices which mountains often do not have.

The estimated sampling rates of all this data is presented in Table 1 within the Related Work section which shows the number of initiates for each district.

An electrocardiogram signal is usually 500 readings a second or $500 \mathrm{~Hz}$. The heart rate is usually sampled at one reading a second or $1 \mathrm{~Hz}$ together with a derived respiration rate at $1 \mathrm{~Hz}$. Plethysmography waves create a waveform from which blood oxygen saturation is derived at $1 \mathrm{~Hz}$. The Artemis platform can also capture movement and skin temperature. However, the collection and analysis of this data constitutes a Big Data problem.

\section{Online Analysis- initiates}

Online analysis will operate in real-time, providing artificial intelligence based real-time predictive analytics and predictive diagnostics for initiates. Algorithms will be tailored to enable support for the general health observation of each initiate. These will incorporate known pathophysiologies evident in physiological data associated with the conditions the initiates are at risk of developing [14].

\section{Result Presentation- initiates}

Real-time and retrospective viewing for diagnosis and physiological data will be provided for use by the traditional leaders and department of health to allow constant management of initiates health and wellness this visualization component.

\section{E. Data Persistency - initiates}

The custodians of the tradition together with the department of health will perform retrospective analysis of initiates during initiation which is often between 3 weeks to 4 weeks. This will require data collected and all analytics produced to be stored within the data storage layer within the designated place chosen by the custodians.

\section{F. Knowledge Extraction- - initiates}

The traditional leader's representatives (medical doctors who have gone through initiation themselves) and department of health's representatives will be allowed to analyse the data collected through utilising the knowledge extraction layer to enable local research if needs be.

\section{G. Deployment and Redeployment- - initiates}

Preliminary clinical rules will produce primary predictive diagnostics and predictive analytics algorithm deployment prior to initiation and updates to clinical rules will continue during the initiation till the end of the initiation. This deployment and redeployment component allows the pathophysiological behaviours determined throughout the knowledge extraction stage to be utilized during the real-time analysis component.

\section{H. Result Presentation to Traditional leaders and Department of Health}

Physiological data and derived data, can be viewed in realtime during initiation and could also be viewed in real time and retrospectively by those at designated data initiation centres (that would be chosen by both Traditional leaders and Department of Health). This would enable the constant observation and health management of initiates by the traditional leaders and health practitioners as per medical support team through the Result Presentation component. 


\section{Data Persistency - data centre}

Aa Artemis Cloud will be operating as a Health Analytics as a Service, initiate data will need to be transmissted to the data center.

\section{J. Knowledge Extraction - data centre}

There will be a need of a more extensive knowledge discovery environment within the designated data center consisting of advanced resources with the ability to increase assimilation knowledge discovery. The inclusive platform may have the ability to support both the monitoring of health and wellbeing during initiation through the integration of the initiates' physiological data, with other relevant clinical data. There will be the potential to buffer this data so that it can be forwarded when there is internet connectivity to do so.

\section{K. Deployment and Redeployment-data centre}

The researchers at the data centre will need the capabilities for deployment and modification of predictive analytics and diagnostics during future initiation periods enabling improved medical support constituted through evidence-based research outcomes gathered from knowledge discovery through research studies.

\section{PRE AND DURING INITIATION MONITORING}

The health of initiates will be monitored prior to initiation to see if they are fit to go through initiation since there are claims that some of the initiates go through initiation while they were involved in alcoholism and drug abuse. During initiation they will also be monitored to detect any changes in their bodies which required urgent attention to avoid death and amputations. Techniques for data acquisition can be used to collect and securely transmit physiological data of initiates to Artemis Cloud before and during initiation, which will allow proper online analyses for diagnostics. The results will be available in support of the medical team and traditional healers for viewing at any time, or if there will be any alerts activated.

\section{CONCLUSION}

This paper propose an architecture test where we send data to the Pawsey High Performance Computing facility in Perth, where an Artemis instance is being developed and there will be enough resources and IT support to access the data as compared to South Africa which currently has no resources to host the Artemis.

Further research can focus on:

1. How illegal centres can be identified in communities.

2. How can the department of education take part in educating the young boys about health issues before they go to initiation.

3. How to use this process as a means to educate boys about STEM through educational activities relating to the technologies used to monitor them.

4. Designating medical practitioners who can work with traditional healers in administering medication interventions to initiates in initiation schools, to allow for completion of initiation in the mountains, and decrease the need to transfers to the hospital.

\section{ACKNOWLEDGMENT}

We would like to acknowledge the House of Traditional Leadership (Eastern Cape Province) for supporting this research. This research is funded in part by McGregor's Research Excellence Chair in Health Informatics.

\section{REFERENCES}

[1] Report on some challenges that lead to deaths and injuries at initiation schools in South $\quad$ Africa. 2017 http://www.crlcommission.org.za/docs/CRL_ORIGINAL_11.pdf

[2] Balaji, S., Patil, M. and McGregor, C., 2017, June. A Cloud Based Big Data Based Online Health Analytics for Rural NICUs and PICUs in India: Opportunities and Challenges. In Computer-Based Medical Systems (CBMS), 2017 IEEE 30th International Symposium on (pp. 385-390). IEEE.

[3] McGregor, C., 2018. Using Constructive Research to Structure the Path to Transdisciplinary Innovation and Its Application for Precision Public Health with Big Data Analytics. Technology Innovation Management Review, 8(8).

[4] Chawla, N.V. and Davis, D.A., 2013. Bringing big data to personalized healthcare: a patient-centered framework. Journal of general internal medicine, 28(3), pp.660-665.

[5] Jagadish, H.V., Gehrke, J., Labrinidis, A., Papakonstantinou, Y., Patel, J.M., Ramakrishnan, R. and Shahabi, C., 2014. Big data and its technical challenges. Communications of the ACM, 57(7), pp.86-94.

[6] Developing Ethical Practices for Public Health Research Data Sharing in South Africa: The Views and Experiences From a Diverse Sample of Research Stakeholders Spencer G. Denny1, Blessing Silaigwana1, Douglas Wassenaar1, Susan Bull2, and Michael Parker2

[7] Keymanthri Moodley,1 Stuart Rennie2. Penile transplantation as an appropriate response to botched traditional circumcisions in South Africa: an argument against

[8] Douglas, M., Maluleke, T.X., Manyaapelo, T. and Pinkney-Atkinson, V., 2018. Opinions and perceptions regarding traditional male circumcision with related deaths and complications. American journal of men's health, 12(2), pp.453-462.

[9] Eastern Cape Provincial Department of Health, 2013; https://pmg.org.za/committee-meeting/16354/.

[10] Ntozini, A.N. and Abdullahi, A.A., 2018. Perceptions of Traditional Male Circumcision among University Male Students at a South African University. Men and Masculinities, 21(2), pp.189-209

[11] McGregor, C, 2019, Service Availability for Real-Time Health Analytics as a Service, University of Ontario Institute of Technology, Oshawa, Ontario, Canada

[12] McGregor, C., 2013, March. A platform for real-time online health analytics during spaceflight. In Aerospace Conference, 2013 IEEE (pp. 1-8). IEEE.

[13] McGregor, C., 2011, June. A cloud computing framework for real-time rural and remote service of critical care. In Computer-Based Medical Systems (CBMS), 2011 24th International Symposium on (pp. 1-6). IEEE.

[14] McGregor, C., 2018. Big Data in Critical Care Using Artemis. In Signal Processing and Machine Learning for Biomedical Big Data (pp. 519531). CRC Press 